\title{
Priorities of positive mental health promotion in the Iranian community: a qualitative study
}

Monir Baradaran Eftekhari ${ }^{1}$, Arash Mirabzadeh ${ }^{2}$, Katayoun Falahat ${ }^{3}$, Homeira Sajjadi ${ }^{4}$, Meroe Vameghi ${ }^{5}$, Gholamreza Ghaedamini Harouni ${ }^{6}$

${ }^{1}$ Ph.D. of Social Determinants of Health, Assistant Professor, Deputy for Research and Technology, Ministry of Health and Medical Education, Tehran, Iran

${ }^{2}$ M.D., Psychiatrist, Professor, Social Determinants of Health Research Center and Department of Psychiatry, University of Social Welfare and Rehabilitation Sciences, Tehran, Iran

${ }^{3} \mathrm{Ph}$.D. Student of Social Determinants of Health, Social Determinants of Health Research Center, University of Social Welfare and Rehabilitation Sciences, Tehran, Iran

${ }^{4}$ M.D., National Board in Community Medicine, Associate Professor, Social Welfare Management Research Center, University of Social Welfare and Rehabilitation Sciences, Tehran, Iran

${ }^{5}$ M.D., Psychiatrist, Associate Professor, Social Welfare Management Research Center, University of Social Welfare and Rehabilitation Sciences, Tehran, Iran

${ }^{6} \mathrm{Ph} . \mathrm{D}$. of Social Welfare, Assistant Professor, Social Welfare Management Research Center, University of Social Welfare and Rehabilitation Sciences, Tehran, Iran

Type of article: Original

\begin{abstract}
Background: Positive Mental Health (PMH) enables people to cope with the common stresses of life and adversity to achieve their full potential and humanity. In many communities, promoting PMH via prioritized interventions has been considered as a key component of public health policies to optimize mental well-being. Objective: To set the priorities of interventional programs of Iranian PMH promotion according to the World Health Organization (WHO) Priority Public Health Condition (PPHC) analytical framework.

Methods: This qualitative study was implemented in 2017 in Tehran, Iran and had two main phases. In a qualitative needs-assessment phase, needs of the community's PMH were collected through eight focus group discussions with a general population aged 30 to 60 years-old. In a priority-setting phase, the priorities of PMH were extracted through an expert panel consisting of mental health professionals and policy makers. Data gathering was implemented based on purposeful sampling according to inclusion criteria. Data were analyzed based on directional content analysis using Dedoose software version 7.6.6.

Results: Fifty-one people and ten mental health professionals and policymakers participated in this study. The process of data analyzing, categorized PMH needs in 4 main categories, 15 subcategories and 46 codes. The four categories were financial security, social security, healthy lifestyle and promoting psychological factors. In the expert panel, first, the indicators of PMH priority setting were determined and then based on the priority public health condition analytical framework of the World Health Organization, the most important of PMH priority in each level was indicated as "creating job positions" in socioeconomic level, "providing proper working conditions" in differential exposure, "promoting practical life skills training" in the differential vulnerability, "easy and affordable access to mental health services" in differential healthcare outcome.

Conclusion: Appropriate policymaking and regulation at national level regarding employment, promoting working conditions, and reducing unemployment, promote community PMH as well as expanding accessible and affordable mental health services in the national healthcare system and empowering the community through providing practical life skill courses.

Keywords: Mental health, Health priorities, Qualitative research, Iran
\end{abstract}

\section{Corresponding author:}

Katayoun Falahat, Social Determinants of Health Research Center, University of Social Welfare and Rehabilitation Sciences, Tehran, Iran. Tel: +98.2181455184 and +98.9126180351, Email: kfalahat@yahoo.com

Received: March 14, 2018, Accepted: May 03, 2018, Published: July 2018

iThenticate screening: April 25, 2018, English editing: May 14, 2018, Quality control: May 15, 2018

This article has been reviewed / commented by four experts

Ethics approval: IR.USWR.REC.1396.204

(C) 2018 The Authors. This is an open access article under the terms of the Creative Commons Attribution-NonCommercialNoDerivs License, which permits use and distribution in any medium, provided the original work is properly cited, the use is non-commercial and no modifications or adaptations are made. 


\section{Introduction}

Today, mental health no longer simply means the absence of mental illness or disorders, and studies around the world place more emphasis on the positive aspects of mental health, such as happiness and wellbeing, and their contribution to mental health promotion $(1,2)$. The importance of this issue is also reflected in World Health Organization (WHO) statements, which argue that mental health is far more than the mere absence of mental disorders $(3,4)$ and generally, these two concepts are not the two ends of one continuum (5). The two-factor model of mental health presented by Keyes also suggests that complete mental health is a function of two separate but correlate axes - one presenting the presence or absence of mental disorders; the other, different levels of Positive Mental Health (PMH) classified as flourishing (high level of PMH), languishing (low level of PMH) and moderate $(6,7)$. Traditionally, PMH is a complex concept that results from the combination of two approaches, namely hedonic or emotional wellbeing and eudemonic approach or psychological wellbeing. The hedonic approach embraces emotional performance, such as happiness and life satisfaction, and the eudemonic approach indicates positive performance such as engagement, fulfillment, sense of meaning, and social wellbeing in personal and social life $(7,8)$. Research shows the increasing importance of $\mathrm{PMH}$ as one of the key components of public health planning and policymaking in many countries (9-12). The advances in pharmacological treatments and extensive psychiatric interventions have not been able to reduce the prevalence and burden of diseases. A systematic review done in 2014 by Steel et al. revealed that worldwide $29.2 \%$ of adults had experienced a mental disorder during their lifetime (lifetime prevalence) and one in five during the previous 12 months (13). In Iran, the prevalence of all types of mental disorder was reported as $23.44 \%$ in 2015 (14). In addition, various studies indicate that low positive mental health is a risk factor for some mental disorders, (especially depression) (15-17). Community-based interventions that promote PMH can have a significant role in protecting and promoting community mental health $(5,18)$. The results of epidemiological studies suggest that patients with mental disorders experience different levels of $\mathrm{PMH}$, and societies considerably vary in the prevalence of flourishing, which indicates that PMH and mental disorders are influenced by different social determinants and each have their own needs and priorities (16, 19-21). Like mental disorders, PMH needs vary in different communities based on the prevailing culture, values and socioeconomic conditions $(5,18)$. Recognizing the prioritized needs is obviously the first step in planning and designing proper interventions to promote community PMH. In 2010, the Priority Public Health Conditions Knowledge Network, one of the networks of the WHO Commission on Social Determinants of Health (CSDH) defined the Priority Public Health Condition (PPHC) analytical framework for determining general health priorities and their complex interactions in communities at five different interventional levels in order to design appropriate interventions, including socioeconomic context and position, differential exposure, differential vulnerability, differential healthcare outcomes and differential consequences (22).

Socioeconomic context and position - related to the structure of the society - has a very significant impact on the type, extent and social distribution of PMH. In 2017, Leah examined the role of socioeconomic discrimination in $\mathrm{PMH}$ and found that increased discrimination reduces PMH (23). Structural social determinants such as education, occupation and income, which shape socioeconomic position, are associated with PMH (24, 25). Differential exposures indicate the relationship between languishing and environmental, psychosocial and behavioral risk factors such as poor lifestyle, obesity, smoking, alcohol consumption and exposure to stressors (26). Differential vulnerability indicates the different vulnerabilities of groups with different socioeconomic position in the face of similar exposures (22). Studies show that personal factors such as health status, gender and income affect PMH in different ways $(27,28)$. A study by Goodwin and his colleagues suggests that help-seeking behaviors are correlated with the level of PMH (29). The lack of PMH knowledge also reduces the use of mental health services and counseling centers at differential healthcare outcome level (30). The loss of job and income, social isolation and the development of mental illnesses such as depression are considered different consequences of a low level of PMH $(12,31,32)$. All studies conducted in Iran on mental health promotion programs have merely looked to diagnose and treatment of mental disorders. They diagnosed mental disorders based on standard protocols and used different drugs for reducing mental disorders (33-35), and there is an obvious lack of studies on mental health promotion with an emphasis on promoting PMH. This study was conducted based on a qualitative method to determine the PMH needs of an Iranian population aged 30 to 60 and understand their priorities based on stakeholders cooperation and recommend helpful interventions to promote $\mathrm{PMH}$ with an emphasis on the WHO five-level PPHC analytical framework. This method has capability to identify PMH needs deeply based on community and experts' opinions. 


\section{Material and Methods}

\subsection{Study Design}

This study was designed and implemented, using directional content analysis. The reason for choosing the qualitative method was the capability of this method to identify and fully comprehend PMH needs from the viewpoint of the community and experts (36). Data were collected through Focus Group Discussion (FGD) within the general population and a panel of mental health professional and policy makers.

\subsection{Participants and selection criteria}

This qualitative study was conducted in Tehran, Iran, in 2017. The participants consisted of two groups: (a) Community members aged 30-60 residing in Tehran, and (b) mental health professionals (psychologists and psychiatrists) and policy makers with at least ten years of research and clinical experience who were willing and able to participate in the study. Considering the purposive nature of sampling with maximum variety, and in order to obtain accounts of different experiences, the FGD participants were selected from a diverse background in terms of age, gender, education, marital status and social class and professionals and policy makers. Participants were also from a diverse background in terms of expertise and work experience. In our study, exclusion criteria for general population was physical or mental disability to participate in FGD and for experts, it was lack of residency in Tehran.

\subsection{Data Collection}

This study had two main stages of qualitative needs assessment and PMH priority setting.

\subsubsection{Qualitative Needs Assessment}

FGD was used for data collection so as to enable an in-depth understanding of the concepts under study through proper interaction with people and the flexibility that the method offers (36). The FGDs continued until data saturation occurred. The FGDs were held in single-gender groups of men and women. Each FGD lasted from 80 to 100 minutes and ended when no further new concepts could be extracted. The number of participants in each FGD ranged from five to eight. Before beginning the group discussions, all the participants were contacted over the phone or in person, and briefed on the general objectives of the study and invited to participate in the group discussions once they had given their verbal consent. The meeting places of the FGDs were selected by the participants and included public spaces such as mosques, municipality community centers, a tailor shop, etc. All the FGDs were conducted by a main researcher in the team (Ph.D. Student) with expertise in qualitative research. At the beginning of each group discussion, the participants were introduced to the facilitator and her colleague (note taker), and briefed on the objectives of the study and then completed the informed consent form for their participation and were assured of the voluntary nature of participation and their right to withdraw from the study whenever they wished. They additionally gave their permission for the audio recording of FGDs. A guide questionnaire was used to conduct and guide the FGDs. It was designed by the research team members in collaboration with an expert panel and using a review of literature according to objectives of the study. Moreover, the guide questionnaire was developed through a pilot FGD participated by five public persons. The final version of the FGD guide consisted of six open questions. Each FGD began with the general question of "Based on your experiences, what are the attributes of a person with positive mental health? Please elaborate". Questions were then posed regarding PMH needs. Proper probing questions or question words (where, when, how and why) and questions such as "What do you mean?" or "Please explain" were used during the interviews. The probing questions were designed to ensure the proper understanding of the concepts discussed by the participants and the discovery of new concepts. The participants were also asked to use objective examples to express their views whenever necessary. A list of the needs and a brief summary were presented to the participants at the end of each group discussion for approval.

\subsubsection{PMH priority setting}

This phase was implemented by the presence of mental health professionals and policymakers. A panel of experts was held with professionals and policy makers based on the inclusion criteria. To set the priorities, based on the Essential National Health Research (ENHRE) approach (34), after presenting a list of all the needs extracted from the qualitative study to the experts, they were asked to:

1) Determine the priority setting indicators based on nominal groups. This means that all of expert panel members introduced some indicators to set the PMH priorities. These indicators were selected based on relevancy, importance, feasibility and probability of success plan.

2) The needs were scored based on a Likert scale from 1 (the least) to 5 (the most) and according to the accepted indicators. Finally, based on the PPHC analytical framework, PMH priorities were placed at their appropriate levels to design appropriate interventions (Figure1). 


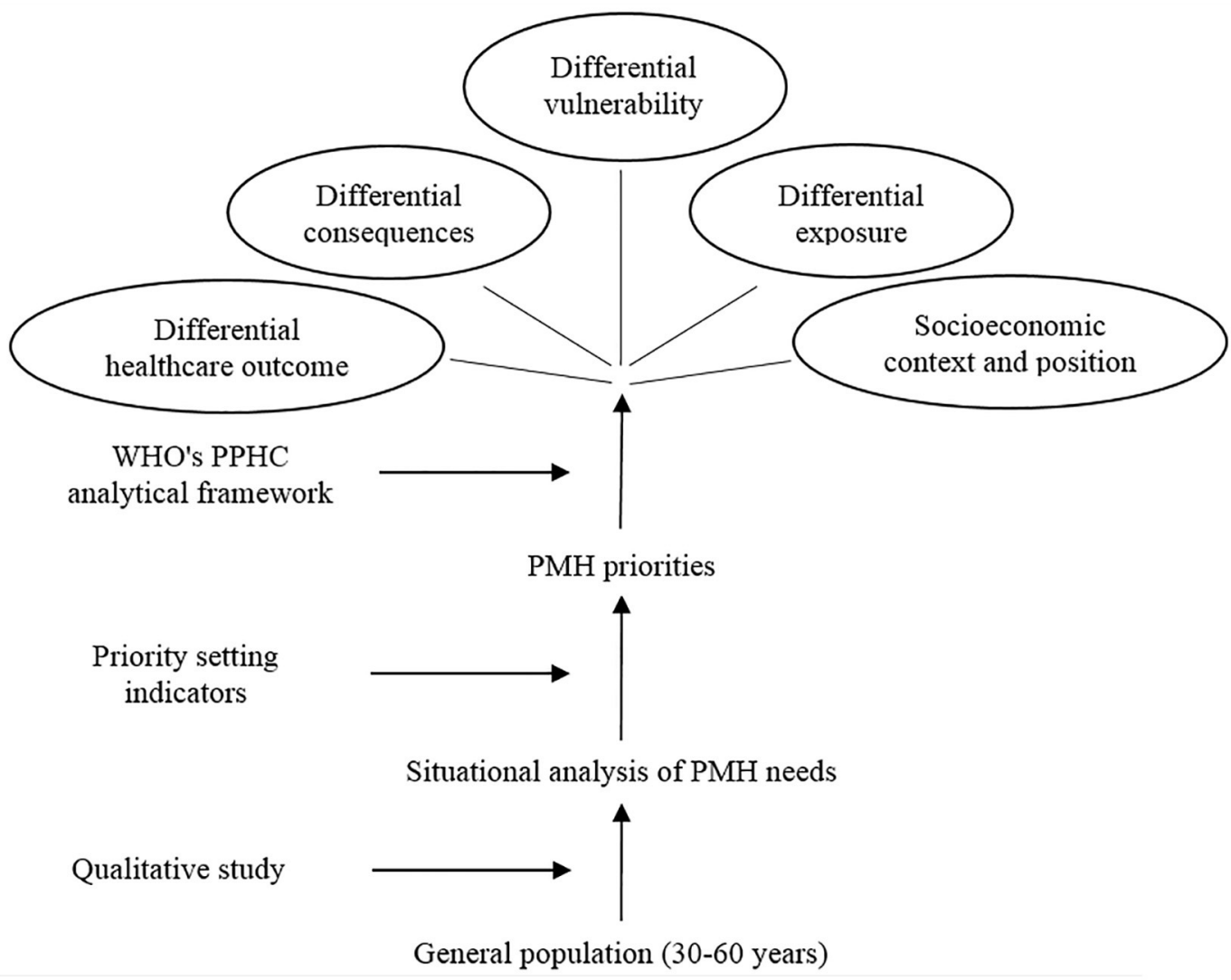

Figure 1. The process of PMH priority setting

\subsection{Data Analysis}

Data analysis was conducted using directed qualitative content analysis in view of the nature of the research subject, objectives and questions. The presence of a theory or research on this approach means that the underlying study variables and the relationships between them can be more easily predicted and it also helps with the initial coding and the establishing of a relationship between the codes (37). Data were analyzed in Dedoose qualitative software version 7.6.6. The data from the group discussions were transcribed verbatim and all field notes and details were added immediately after the end of each session, and were analyzed concurrently with data collection. To ensure accuracy and precision, the transcribed data were compared against the recordings. All the transcribed group discussions were typed in MS Word ${ }^{\mathrm{TM}}$ and imported to software. The group discussions were read line by line and proper codes were assigned by the software. The meaning units were extracted based on the study framework and according to the PMH needs. After extracting the codes, the subcategories were formed according to similarities and then a review was carried out to obtain the list of the needs. The researchers' long-term involvement with the subject of the study for the purpose of data collection, their interaction with the participants, the frequent review of the data and ensuring data saturation were steps that helped the different aspects of study trustworthiness such as credibility, member check, dependability, consistency and transferability the validity. The participants were selected from a diverse background in order to ensure data credibility. All the findings from each FGD were shared with the participants at the end of the session and were controlled again for respondent validation. To ensure the dependability of the data, all FGDs were immediately transcribed and all their non-verbal content and their context were added to the transcriptions. The stages of data transcription and analysis were carried out by two experts from the research team for peer checking. The research team members ensured team consistency during the process of data analysis. The stages of the research were recorded in detail for use by other researchers in order to ensure data transferability.

\subsection{Ethical Considerations}

The ethical considerations of this research included obtaining informed consent from the participants, assuring them of the voluntary nature of participation in the study and their right to withdraw from it at any time and ensuring the confidentiality of their personal information and data discussed in the sessions. The study protocol was approved by 
http://www.ephysician.ir

the Ethics Committee of the University of Social Welfare and Rehabilitation Sciences under the code IR.USWR.REC.1396.204.

\section{Results}

In this section, the results of the qualitative needs assessment and priority setting of PMH needs are categorized as follows:

\subsection{Qualitative Needs Assessment}

Eight FGDs were held with the general public (men and women, separately) for the qualitative needs assessment. Considering study inclusion criteria, 51 people participated in the FGDs, of which $45.8 \%$ were female. The participants had a mean age of 46.1 years. Table 1 presents the demographic details of these participants. After data analyzing and extracting the needs, the needs were incorporated into the 4 categories, 15 subcategories and 46 codes. The four categories were financial security, social security, healthy lifestyle and promoting psychological factors. All derived categories, subcategories and codes are presented in Table 2.

Table 1. Characteristics of participants in FGDs

\begin{tabular}{|l|l|l|l|l|l|l|l|l|}
\hline Sex (n) & $\begin{array}{l}\text { Age (year); } \\
\text { Mean } \pm \text { SD }\end{array}$ & Education & \multicolumn{2}{|l|}{ Employment status } & \multicolumn{2}{l|}{ Marital status } \\
\cline { 3 - 9 } & & $\begin{array}{l}\text { High School } \\
\text { Diploma or lower }\end{array}$ & $\begin{array}{l}\text { Higher than High } \\
\text { School Diploma }\end{array}$ & Employed & Housewife & Unemployed & Married & Single \\
\hline Female (29) & $45.3 \pm 8$ & 18 & 11 & 14 & 11 & 4 & 24 & 5 \\
\hline Male (22) & $46.9 \pm 8.6$ & 6 & 16 & 18 & 0 & 4 & 16 & 6 \\
\hline
\end{tabular}

Table 2. Extracted codes and subcategories of PMH Needs in participants 30-60 years-old

\begin{tabular}{|c|c|c|}
\hline Category & Subcategory & Code \\
\hline \multirow[t]{4}{*}{ Financial security } & $\begin{array}{l}\text { Stability in economic } \\
\text { legislation }\end{array}$ & $\begin{array}{l}\text { 1) Multiple labor law; 2) Instability in labor laws; 3) Instability in } \\
\text { economic situation }\end{array}$ \\
\hline & Creating job positions & $\begin{array}{l}\text { 1) Employment and purposeful life; 2) Employment and motivation; 3) } \\
\text { Decrease living stress source; 4) Increase personal capabilities; 5) Create } \\
\text { identity }\end{array}$ \\
\hline & $\begin{array}{l}\text { Providing proper working } \\
\text { condition }\end{array}$ & 1) Occupational stress; 2) Job satisfaction; 3) Job relationship \\
\hline & $\begin{array}{l}\text { The elimination of gender } \\
\text { discrimination in working } \\
\text { condition }\end{array}$ & $\begin{array}{l}\text { 1) Experience lower salary in women; 2) Experience higher stress in } \\
\text { women; 3) Experience lower occupational position in women }\end{array}$ \\
\hline \multirow[t]{4}{*}{ Social security } & Securing social justice & $\begin{array}{l}\text { 1) Comprehensive legislation for different social groups; 2) Equally } \\
\text { access to social services; 3) Competitive educational system }\end{array}$ \\
\hline & Universal insurance coverage & 1) Disability insurance; 2) Unemployment benefits; 3) Retirement pension \\
\hline & $\begin{array}{l}\text { Maintaining and promoting } \\
\text { sources of social support }\end{array}$ & $\begin{array}{l}\text { 1) Family support; 2) Social relations; 3) Support from governmental } \\
\text { sectors }\end{array}$ \\
\hline & $\begin{array}{l}\text { Facilitating of marriage and } \\
\text { marital life }\end{array}$ & 1) Family upbringing; 2) Emotional support; 3) Interpersonal relationships \\
\hline \multirow[t]{4}{*}{ Healthy life style } & $\begin{array}{l}\text { Healthy physical and social } \\
\text { environment }\end{array}$ & $\begin{array}{l}\text { 1) Safe physical environment; 2) Healthy neighborhood (physically \& } \\
\text { socially); 3) Urban dilemmas }\end{array}$ \\
\hline & $\begin{array}{l}\text { Preventing of high-risk } \\
\text { behaviors }\end{array}$ & $\begin{array}{l}\text { 1) Smoking / drug use / drinking; 2) Disruption of interpersonal } \\
\text { relationships and family; 3) Lack of family solidarity }\end{array}$ \\
\hline & Appropriate physical activity & 1) Reduced daily stress; 2) Hopefulness \\
\hline & $\begin{array}{l}\text { Proper use of internet and } \\
\text { the media }\end{array}$ & $\begin{array}{l}\text { 1) In proper source of information; 2) Creating stress; 3) Offering wrong } \\
\text { model in family roles; 4) Weakening interpersonal relationships }\end{array}$ \\
\hline \multirow{3}{*}{$\begin{array}{l}\text { Promoting } \\
\text { psychological } \\
\text { factor }\end{array}$} & Promoting practical life skills & 1) Stress management; 2) Anger control; 3) Parenting skills \\
\hline & $\begin{array}{l}\text { Promoting PMH knowledge } \\
\text { and literacy }\end{array}$ & 1) PMH knowledge and literacy; 2) PMH services; 3) Community stigma \\
\hline & $\begin{array}{l}\text { Easy and affordable access to } \\
\text { mental health services }\end{array}$ & $\begin{array}{l}\text { 1) Access to mental health services; 2) Providing culturally / linguistically } \\
\text { mental health services }\end{array}$ \\
\hline
\end{tabular}




\subsubsection{Financial security}

In this category, 4 subcategories, 14 codes and 154 meaning units were extracted. According to participants' experiences, four subcategories were "stability in economic legislation", "creating job positions", "providing proper working conditions" and "eliminating gender discrimination in working conditions" as follows:

a- Stability in economic legislation

One of the most important issues addressed, was community political and economic stability, and all participants focused on the important role of this subcategory considering existing problems and difficulties in society derived from this instability. Political instability, sanctions, and financial insecurity, inflation, etc. can affect people's PMH. Participants also referred to the negative effects of economic instability and its consequence on people especially demolishing humanitarian considerations that directly affect PMH. "Multiple law changes can create stressful situations and we can not plan appropriate programs for our lives" (educated married man)

$\boldsymbol{b}$ - Creating job positions

Establishing appropriate rules in order to create proper employment has positive effects on community mental health. In all FGDs, the participants presented that there are a lack of job positions in the community. They stressed that being employed and having a proper job and income is vital in PMH promotion. The majority of participants noted that, qualifications such as motivation, purposefulness plus financial security in employed people, decrease living stress and ensure PMH. "Unemployment is very bad for everyone and can cause hardship in life. In most situations, an unemployed person loses his control and turns to illegal actions" (Uneducated single man). Most female participants stated that nowadays, many women play two different roles as an employee and homemaker. They earn less income than men do and have less time for their important second role in the family. Tolerating such stresses can have a negative effect on $\mathrm{PMH}$, although some of the participants noted that having a job can create identity and increase personal capabilities.

c- Providing proper working conditions

Proper working conditions, such as job security (income), occupational stress (workload difficulties and work hours), job satisfaction and the physical workplace are effective on people's $\mathrm{PMH}$, mentioned by all participants "Job satisfaction is very important. I mean satisfaction with everything; for example, the type of job, income, work environment, colleagues, and job security" (an educated woman)

d-Eliminating gender discrimination in working conditions

Based on participants' views (most females and less males) women experience lower salaries and lower positions, despite their efficiency and proper leadership in work places. Manager positions are mostly filled by men. Participants expressed people who are exposed to such conditions, tolerate more stress and pressure. "Men are more likely to be bosses in the workplace, they get higher salaries, but women, although they are more efficient, are always in a lower position." (An employed educated married woman)

3.1.2. Social security

In this category, 4 subcategories, 12 codes and 134 meaning units were extracted. According to participants' views, four subcategories were "Securing social justice", "Universal insurance coverage", "Maintaining and promoting sources of social support" and "Facilitating of marriage and marital life" defined as follows:

a- Securing social justice

The majority of participants stated it is needed to have comprehensive legislation for all the social groups in order to access equal and proper social services. Although they focused especially on different aspects of existing social and economic injustice in people with different socioeconomic positions, they considered most of the existing services are not provided properly, responsively and based on community needs. These services and their policies not only solve the problems but also define different kinds of social injustice. "Now, for some marginalized people, there are inadequate mental health promotion services, and it is a need in this social group despite their many various other needs..." (An educated single man). "If social justice prevails in the economy, the poor economic situation will disappear and positive mental health will improve." (An uneducated married woman). In general, social justice should be embedded in the dominant political, social and cultural structure of the society in all its components, and its presence contributes significantly to the promotion of $\mathrm{PMH}$. One of the important social aspect of injustice in the community considered by participants was the competitive educational system of the country such as university entrance exams that bring many inequalities affecting mental health status. "Many people who want to go to university have tough competition and this is very stressful. Although those who pass the entrance exam are very successful in their university and job, they have lots of mental problems." (An educated married woman)

b- Universal insurance coverage

Universal insurance coverage includes health insurance, disability insurance, unemployment benefits and retirement pension. All participants stated that having fixed income and insurance (permanent job) is one the most important necessities in everyone's life. Social security remarkably, has relationship with PMH. "People with insurance are 
less worried about their future and are sure that there is at least something to keep them going, and this makes them feel more comfortable. In my opinion, insurance and pension are essential needs". (an educated woman)

c- Maintaining and promoting sources of social support

Inadequate social support in different levels including family members, social relations and governmental sectors has a key role in reducing PMH. Based on participants' views, the family is the main source of support and affects $\mathrm{PMH}$ in different manners, including family upbringing, emotional and spiritual support, and interpersonal relationships. Kindness, friendship and morality empower a person and can resolve many mental and physical problems. Some participants highlighted the positive role of family support in elders and promotion of their PMH level. "Being raised in a family environment is very important and people without family show this deficit in all of their aspects of life. They can not come to terms with this deprivation" (Men's educated group). "It is important to get support on all levels, from family to community". (Uneducated woman)

d- Facilitation of marriage and marital life

All the participants unanimously agreed that marriage has positive affect on PMH regardless of its quality. Humans have a social nature and depend on others, and marriage eliminates this demand. Marriage is accompanied with skills such as management, responsibility, reliably and trust and purposefulness. According to participants' views, marriage at the right time is sacred, a matter greatly emphasized in the Islamic religion and Iranian culture. "Because he is essentially committed to a number of other people, the married person is more reliable. I place greater trust in married people. They may have more problems, but a married person is more responsible and has better mental health." (A married educated man). Public participants also pointed to the influence of marriage quality on PMH. A close interpersonal relationship, proper mutual understanding and interaction, and finally, marital satisfaction increase the level of PMH. Contrarily, a poor marriage, hardships and ultimately divorce impair PMH. The majority of the public participants emphasized the role of family and marital relationship in PMH. "In my opinion, marriage, irrespective of its outcome, affects $\mathrm{PMH}$, since it leads to evolvement -especially in the Iranian culture, where marriage and family formation have a high social value" (A married educated woman)

3.1.3. Healthy lifestyle

According to the views of the general population, the needs in this category which influence PMH were summarized in 12 codes, 247 meaning units and four subcategories as "healthy physical and social environment", "prevention of high-risk behaviors", "appropriate physical activity" and "proper use of the internet and the media".

a- Healthy physical and social environment

Providing a healthy and safe physical environment in both the home and the neighborhood was mentioned by all the participants. Traffic, air pollution, population density, dirty and polluted places and many other urbanization problems were considered as a usual source of daily stress experiences by urban people. Controlling these stresses increases the level of PMH. The majority of participants highlighted the importance of accessible and affordable recreation places to revitalize and achieve peace and happiness such as modern parks in the community, especially for youths. They also mentioned the influence of different negative aspects of social condition of the environment (home, neighborhoods and the community) such as lack of public safety, crime and violence on people, trust, relationships and social life. "Where you live is a very important matter -in the town or in the village. Different expectations, the traffic, noise and even air pollution affect your positive mental health very much." (an educated man). "...Urban people spend hours in traffic and are under much pressure and breathe polluted air and so always live in pressure and stress..." (a single educated woman )

b- Prevention of high-risk behaviors

High-risk behaviors such as drinking, smoking and drug use is detrimental to PMH. Tendency toward high-risk behaviors is a result of low and poor PMH in the population. According to participants' experience, these behaviors considered as stigma in the community, have effects on family relationships, family stability, and personal responsibility, relationship and life. "Drug abuse can reduce responsibility in the family and has a lot of side effects on family solidarity" (an uneducated woman)

c- Appropriate physical activity

Most of the participants pointed out that physical activity reduced daily stress and is very important in PMH promotion. Physical activity is accompanied with stress management skill, thinking positively, happiness and solving problem skills. The participants insisted in positive effects of regular daily exercise in human psychological aspects. Lack of proper polices and interventions in order to develop facilities and acceptable places have been considered as the main important need in this issue. "Lack of physical activity consequences in isolation and depression." (a married uneducated woman)

$\boldsymbol{d}$-Proper use of the internet and the media

The internet is a major source of information for people but is not properly monitored. Creating stress, offering the wrong model in family roles, and weakening interpersonal relationships are side effects of inappropriate use of 
virtual networks affecting PMH. "Everybody forgets real life. People do not know each other. They cannot talk, communicate or show their feelings. They just know your ID and stickers and show their emotion by sending stickers." (a married educated man)

\subsubsection{Promoting psychological factors}

Based on general population experiences, the needs in this category that influence PMH were summarized in 3 subcategories, 8 codes and 98 meaning units. The subcategories are "promoting practical life skills", "promoting PMH knowledge and literacy" and "easy and affordable access to mental health services" as mentioned below.

\section{a- Promoting practical life skills}

Training such as stress management, parenting, and anger control etc., is an inseparable part of PMH, as mentioned by participants. Participants agreed life skills training is necessary; it helps people cope with stressful situations, shows capabilities and promotes their PMH level. "In coping with difficulties, people must try to find the best solution, and learning of different stress management skills can help them." (educated men`s group).

\section{$\boldsymbol{b}$ - Promoting PMH knowledge and literacy}

$\mathrm{PMH}$ literacy is an essential factor to PMH promotion by encouraging people to use PMH services more properly and reducing community stigma.

c- Easy and affordable access to mental health services

Access to mental health services and its quality were mentioned by most of the participants. Providing culturally and linguistically mental health services can reduce the complaints of low levels of $\mathrm{PMH}$, such as preventing depression and social isolation. "Most of the time, a mental health counselor is much better and more practical at solving your problem, reassuring you and showing the right path; but not everyone can go to a counselor, because it's very costly" (an educated single woman). "it is important, these services match with people's beliefs and values" (an educated married man)

\subsection{PMH Priority Setting}

To determine the priorities, based on inclusion criteria, ten eligible mental health professionals and policy makers (six women) with an average 21.6 years of work experience attended the panel. Table 3 presents the demographic details of these participants. In this panel, a list of needs was presented. The experts discussed each need and its level and then, the indicators for priority setting were determined based on participants' viewpoints using nominal group and brain storming (Table 4). According to the determined priority setting indicators and score obtained for each need (based on a Likert score from 1 to 5), the maximum and minimum scores were 338 (creating job positions) and 78 (appropriate physical activity) respectively. The list of needs and the first PMH priority of adults aged 30-60 based on a PPHC framework of the WHO was shown in Table 5.

Table 3. Characteristics of participants of the expert panel

\begin{tabular}{|l|l|l|l|l|}
\hline Sex (n) & \multirow{2}{*}{ Age; Mean \pm SD } & Experts & Work experience \\
\cline { 3 - 4 } & & Professionals & Policy makers & \\
\hline Female (6) & $50.8 \pm 5.5$ & 4 & 2 & 24.8 \\
\hline Male (4) & $56.1 \pm 8.2$ & 3 & 1 & 18.4 \\
\hline
\end{tabular}

Table 4. The indicators of priority setting for PMH in Iranian society

\begin{tabular}{|l|l|}
\hline Indicator & Indicator Components \\
\hline Appropriateness & Moral and ethical needs appropriate for the Iranian society \\
\hline Relevance & The necessity of the problem \\
\cline { 2 - 2 } & The severity and significance of the problem \\
\cline { 2 - 2 } & The demand of the community \\
\cline { 2 - 2 } & Trend of the problem \\
\hline Chances of Success & Ability to implement appropriate interventions \\
\hline Chances of Success & Being economical (cost justification) \\
\hline
\end{tabular}


http://www.ephysician.ir

Table 5. List of needs and first priority of PMH in adults aged 30-60 according to PPHC framework of the WHO

\begin{tabular}{|c|c|c|}
\hline PPHC Interventional Level & List of Needs & First Priority in Each Level \\
\hline \multirow{3}{*}{$\begin{array}{l}\text { Socioeconomic context and } \\
\text { position }\end{array}$} & Stability in economic legislation & \multirow[t]{3}{*}{ Creating job positions } \\
\hline & Creating job positions & \\
\hline & Securing social justice & \\
\hline \multirow[t]{7}{*}{ Differential exposure } & Providing proper working condition & \multirow[t]{7}{*}{ Providing proper working condition } \\
\hline & Universal insurance coverage & \\
\hline & Healthy physical and social environment & \\
\hline & Appropriate physical activity & \\
\hline & Preventing of high-risk behaviors & \\
\hline & Proper use of internet and the media & \\
\hline & $\begin{array}{l}\text { Maintaining and promoting sources of } \\
\text { social support }\end{array}$ & \\
\hline \multirow[t]{4}{*}{ Differential vulnerability } & Promoting practical life skills & \multirow[t]{4}{*}{ Promoting practical life skills } \\
\hline & $\begin{array}{l}\text { Elimination of gender discrimination in } \\
\text { working condition }\end{array}$ & \\
\hline & Facilitating of marriage and marital life & \\
\hline & Promoting PMH knowledge and literacy & \\
\hline $\begin{array}{l}\text { Differential health care } \\
\text { outcome }\end{array}$ & $\begin{array}{l}\text { Easy and affordable access to mental health } \\
\text { services }\end{array}$ & $\begin{array}{l}\text { Easy and affordable access to mental } \\
\text { health services }\end{array}$ \\
\hline Differential consequences & & \\
\hline
\end{tabular}

\section{Discussion}

The results of this study show that PMH priorities include creating job positions, providing proper working conditions, promoting practical life skills, and easy and affordable access to mental health services. To date, several studies have examined the positive role of employment in PMH. A study by Giuntoli (2012) in the UK demonstrated the important role of employment in PMH and showed how unemployment affects mental well-being (38). In another study conducted by Cheng and her colleague in Great Britain, it was shown that occupational prestige was significantly associated with high mental wellbeing (39). Various studies around the world have examined the significance of employment, job quality, job security, working capacity, workplace stress and working environment on PMH (31, 40-43). Another important PMH need prioritized at the differential exposure level is providing proper working conditions. Given the participants' age group in this study is defined as adulthood, giving the first two priorities to occupation-related needs at both national and community levels demonstrates the importance of employment and its related conditions in this age group (44-46). Regardless of other work conditions, work hours play an important role in PMH. A study by Li et al. (2014) in China showed that long working hours, dissatisfaction with income, type of occupation and greater use of social support resources are associated with PMH (32). In view of the high priority given to occupation and the improvement of work conditions, it is important for the authorities to make efforts to create more jobs, improve working conditions and raise wages, and take effective steps to develop new and improved measures on the existing laws at the macro level, and adopt effective enforcement policies. Promoting practical life skills, such as anger management, stress management, parenting and time and financial resource management, comprised another priority set at the differential vulnerability level. This qualitativelyextracted priority demonstrates the importance of learning practical life skills in promoting mental health and thereby PMH (34). Considering that stress is the most effective predictor of mental health, the use of problemoriented coping strategies plays a significant role in promoting mental health (34). Another prioritized need for promoting PMH is easy and affordable access to mental health services. The effectiveness of the interventions designed to build these mental health counseling centers ultimately determines how the community PMH is improved (5). Integrating mental healthcare with PMH approach in a national primary health care (PHC) system such as establishing mental health promotion units in healthcare centers is a positive step toward achieving this important target. In Iran, mental health counseling services have become more easily accessible in recent years, but since mental health-related issues are stigmatized in society, building a culture that seeks these services is essential, and relevant organizations such as the media, play an important role in promoting the use of these services. A review of the results suggests that some of the priorities set for improving PMH in the Iranian community differ from what is generally observed in other communities; for example, physical activity and proper eating habits, which are prioritized needs in studies, especially ones conducted in developed countries $(26,47)$, were not considered very important by the Iranian participants and were not mentioned as a priority. Moreover, in Iranian society, the issues 
of social security and insurance coverage are important PMH needs that were not much emphasized in other studies. Considering the PPHC framework of the WHO, which has five interventional levels, the finding indicated no priority in the fifth level of this framework, which covers rehabilitation and harm reduction interventions. In 2010, the results of a study by the WHO Regional Office for the Eastern Mediterranean explained that interventions at these five mentioned levels help reduce high-risk behaviors such as smoking and unsafe sex, provide better access to primary health care services and reduce social problems such as school dropout and domestic violence, which can then result in an improved PMH (22).

\section{Limitations}

This study contains limitations. Using qualitative research methodology, the generalization and representativeness of the results are not possible for people in similar conditions and only its process, methodology and the model employed in the study can be applied in other populations. The results of this study require more research attempts towards being applicable to the whole Iranian population with its multi ethnicity context. Moreover, since only the middle-aged (30-60 years old) were examined in this study, the priorities extracted cannot be generalized to younger and older adults.

\section{Conclusions}

In this study, we introduced the needs of PMH and its priorities according to the views of Iranian participants. In summary, the PMH priority setting revealed the importance of employment, working conditions and environment on PMH in Iranian middle age. Besides this, it showed that applying a qualitative approach is an effective step toward extracting PMH needs and priorities with the participation of people and experts. Furthermore, applying PPHC analytical framework for classification of the needs helps the policymakers to develop interventional programs at national, community and individual level.

\section{Acknowledgments:}

The authors appreciate all the participants who contributed to the study and shared their experiences regarding positive mental health. This study was derived from a $\mathrm{PhD}$ thesis regarding positive mental health and its social determinants and priorities approved by the research council of the University of Social Welfare and Rehabilitation Sciences.

\section{Conflict of Interest:}

There is no conflict of interest to be declared.

Authors' contributions:

All authors contributed to this project and article equally. All authors read and approved the final manuscript.

\section{References:}

1) Laidlaw A, McLellan J, Ozakinci G. Understanding undergraduate student perceptions of mental health, mental well-being and help-seeking behaviour. Stud High Educ. 2016; 41(12): 2156-68. doi: 10.1080/03075079.2015.1026890.

2) Stein DJ. Positive mental health: a note of caution. World psychiatry: official journal of the World Psychiatric Association (WPA). 2012; 11(2): 107-9. doi: 10.1016/j.wpsyc.2012.05.023. PMID: 22654942, PMCID: PMC3363386.

3) WHO. Strengthening mental health promotion: mental health is not just the absence of mental disorder. Fact sheet no 220. Geneva: World Health Organization; 2001.

4) WHO. Investing in mental health. Geneva: World Health Organization; 2003.

5) Friedli L. Mental health, resilience and inequalities. WHO Regional Office for Europe Copenhagen; 2009.

6) Keyes CL. To flourish or not: positive mental health and all-cause mortality. Am J Public Health. 2012; 102(11): 2164-72. PMID: 22994191, PMCID: PMC3477942.

7) Keyes CL. Mental health as a complete state: How the salutogenic perspective completes the picture. Bridging Occupational, Organizational and Public Health. Springer. 2014: 179-92. doi: 10.1007/978-94007-5640-3_11.

8) Keyes CL, Dhingra SS, Simoes EJ. Change in level of positive mental health as a predictor of future risk of mental illness. Am J Public Health. 2010; 100(12): 2366. doi: 10.2105/AJPH.2010.192245. PMID: 20966364, PMCID: PMC2978199. 
9) Khawaja NG, Ibrahim O, Schweitzer RD. Mental Wellbeing of Students from Refugee and Migrant Backgrounds: The Mediating Role of Resilience. Sch Ment Health. 2017: 1-10. doi: 10.1007/s12310-0179215-6.

10) Orpana H, Vachon J, Dykxhoorn J, McRae L, Jayaraman G. Monitoring positive mental health and its determinants in Canada: the development of the Positive Mental Health Surveillance Indicator Framework. Health Promot Chronic Dis Prev Can. 2016; 36(1): 1-10. PMID: 26789022, PMCID: PMC4939463.

11) Jivraj S, Nazroo J. Determinants of socioeconomic inequalities in subjective well-being in later life: a cross-country comparison in England and the USA. Qual Life Res. 2014; 23(9): 2545-58. doi: 10.1007/s11136-014-0694-8. PMID: 24789666.

12) Dreger S, Buck C, Bolte G. Material, psychosocial and sociodemographic determinants are associated with positive mental health in Europe: a cross-sectional study. BMJ Open. 2014; 4(5): e005095. doi: 10.1136/bmjopen-2014-005095. PMID: 24871540, PMCID: PMC4039806.

13) Steel Z, Marnane C, Iranpour C, Chey T, Jackson JW, Patel V, et al. The global prevalence of common mental disorders: a systematic review and meta-analysis 1980-2013. Int j Epidemiol. 2014: 43(2): 478-93. doi: 10.1093/ije/dyu038. PMID: 24648481, PMCID: PMC3997379.

14) Noorbala AA, Faghihzadeh S, Kamali K, Bagheri Yazdi SA, Hajebi A, Mousavi MT, et al. Mental Health Survey of the Iranian Adult Population in 2015. Arch Iran Med. 2017; 20(3): 128-34. doi: 0172003/AIM.003. PMID: 28287805.

15) Keyes CL, Dhingra SS, Simoes EJ. Change in level of positive mental health as a predictor of future risk of mental illness. Am J Public Health. 2010; 100(12): 2366-71. doi: 10.2105/ajph.2010.192245. PMID: 20966364, PMCID: PMC2978199.

16) Keyes CL, Eisenberg D, Perry GS, Dube SR, Kroenke K, Dhingra SS. The relationship of level of positive mental health with current mentaldisorders in predicting suicidal behavior and academic impairment in college students. J Am Coll Health. 2012; 60(2): 126-33. doi: 10.1080/07448481.2011.608393. PMID: 22316409.

17) Wood AM, Joseph S. The absence of positive psychological (eudemonic) well-being as a risk factor for depression: A ten year cohort study. J Affect Disor. 2010; 122(3): 213-7. PMID: 19706357, doi: 10.1016/j.jad.2009.06.032.

18) Barry MM. Addressing the determinants of positive mental health: concepts, evidence and practice. Int J Ment Health Promot. 2009; 11(3): 4-17. doi: 10.1080/14623730.2009.9721788.

19) Huppert FA, So TT. Flourishing across Europe: Application of a new conceptual framework for defining well-being. Soc Indic Res. 2013; 110(3): 837-61. PMID: 23329863, PMCID: PMC3545194. doi: 10.1007/s11205-011-9966-7.

20) Seow LSE, Vaingankar JA, Abdin E, Sambasivam R, Jeyagurunathan A, Pang S, et al. Positive mental health in outpatients with affective disorders: Associations with life satisfaction and general functioning. J Affect Disord. 2016; 190: 499-507. doi: 10.1016/j.jad.2015.10.021. PMID: 26561940.

21) Stewart-Brown S, Samaraweera P, Taggart F, Kandala NB, Stranges S. Socio-economic gradients and mental health: implications for public health. Br J Psychiatry. 2015; 52(1): 34-9. doi: 10.1111/jpc.1301.

22) Blas E, Kurup AS. Equity, social determinants and public health programmes: World Health Organization; 2010. Avaiable from: http://whqlibdoc.who.int/publications/2010/9789241563970_eng.pdf.

23) Leah V. Mental well-being and independence for older people. Nurs Older People. 2017; 29(2): 11. doi: 10.7748/nop.29.2.11.s11. PMID: 28244348.

24) Gilmour H. Positive mental health and mental illness. Health Rep. 2014; 25(9): 3-9. PMID: 25229895.

25) Putz R, Clarke A, Hamborg T, Franco OH. What factors are associated with a validated measure of mental wellbeing in the general population in Coventry? A stratified random cross sectional survey. J Epidemiol Community Health. 2011; 65: A14. doi: 10.1136/jech.2011.143586.31.

26) Stranges S, Samaraweera PC, Taggart FM, Stewart-Brown S. Major behavioural risk factors and mental wellbeing in the general population: a cross sectional analysis of the Health Survey for England J Epidemiol Community Health. 2014; 68: A69. doi: 10.1136/jech-2014-204726.150.

27) Ab-Murat N, Mason L, Kadir RA, Yusoff N. Self-perceived mental well-being amongst Malaysian dentists. Int J Occup Saf Ergon. 2017: 1-7. doi: 10.1080/10803548.2016.1268869. PMID: 28010176.

28) Gestsdottir S, Arnarsson A, Magnusson K, Arngrimson SA, Sveinsson T, Johannsson E. Gender differences in development of mental well-being from adolescence to young adulthood: An eight-year follow-up study. Scand J Public Health. 2015; 43(3): 269-75. doi: 10.1177/1403494815569864. PMID: 25712030 . 
29) Goodwin J, Behan L, Kelly P, McCarthy K, Horgan A. Help-seeking behaviors and mental well-being of first year undergraduate university students. Psychiatry Res. 2016; 246: 129-35. doi: 10.1016/j.psychres.2016.09.015. PMID: 27693865.

30) De Cates A, Stranges S, Blake A, Weich S. Mental well-being: An important outcome for mental health services? Br J Psychiatry. 2015; 207(3): 195-7. doi: 10.1192/bjp.bp.114.158329. PMID: 26329562.

31) De Moortel D, Vandenheede H, Muntaner C, Vanroelen C. Structural and intermediary determinants of social inequalities in the mental well-being of European workers: A relational approach. BMC Public Health. 2014; 14(1): 938. doi: 10.1186/1471-2458-14-938. PMID: 25201291, PMCID: PMC4175570.

32) Li J, Chang SS, Yip PSF, Li J, Jordan LP, Tang YG, et al. Mental wellbeing amongst younger and older migrant workers in comparison to their urban counterparts in Guangzhou city, China: a cross-sectional study. BMC Public Health. 2014; 14: 1280. doi: 10.1186/1471-2458-14-1280. PMID: 25510169, PMCID: PMC4301935.

33) Owlia P, Eftekhari MB, Forouzan AS, Bahreini F, Farahani M, Ghanei M. Health research priority setting in Iran: Introduction to a bottom up approach. J Res Medical Sci. 2011; 16(5): 691-8. PMID: 22091294, PMCID: PMC3214383.

34) Eftekhari MB, Forouzan AS, Mirabzadeh A, Sajadi H, Dejman M, Rafiee H, et al. Mental Health Priorities in Iranian Women: Overview of Social Determinants of Mental Health. Iran J Ssychiatry. 2014; 9(4): 241 7. PMID: 25792993, PMCID: PMC4361828.

35) Rahimi-Movaghar A, Amin-Esmaeili M, Hefazi M, Rafiey H, Shariat SV, Sharifi V, et al. National Priority Setting for Mental Health in Iran. Iran J Psychiatry \& Clin Psychol. 2014; 20(3): 189-200.

36) Holloway I, Galvin K. Qualitative research in nursing and healthcare: John Wiley \& Sons; 2016.

37) Hsieh HF, Shannon SE. Three approaches to qualitative content analysis. Qual Health Res. 2005; 15(9): 1277-88. doi: 10.1177/1049732305276687. PMID: 16204405.

38) Giuntoli G. Going for 'the full monty'? Unemployment and mental wellbeing during times of recession. Ment Health Today. 2012: 24-7. PMID: 23304883.

39) Cheng H, Furnham A. The Associations Between Parental Socio-Economic Conditions, Childhood Intelligence, Adult Personality Traits, Social Status and Mental Well-Being. Soc Indic Res. 2014; 117(2): 653-64. doi: 10.1007/s11205-013-0364-1.

40) Bertilsson M, Vaez M, Waern M, Ahlborg G Jr., Hensing G. A Prospective study on self-assessed mental well-being and work capacity as determinants of all-Ccause sickness absence. J Occup Rehabil. 2015; 25(1): 52-64. doi: 10.1007/s10926-014-9518-5. PMID: 24898192.

41) De Moortel D, Thévenon O, De Witte H, Vanroelen C. Working Hours Mismatch, Macroeconomic Changes, and Mental Well-being in Europe. J Health Soc Behav. 2017; 58(2): 217-31. doi: 10.1177/0022146517706532. PMID: 28661780.

42) Page K, Milner AJ, Martin A, Turrell G, Giles-Corti B, LaMontagne AD. Workplace Stress What Is the Role of Positive Mental Health? J Occup Environ Med. 2014; 56(8): 814-9. doi: 10.1097/jom.0000000000000230. PMID: 25099407.

43) Vulkan P, Saloniemi A, Svalund J, Vaisanen A. Job Insecurity and Mental Well-Being in Finland, Norway, and Sweden. Nord J Working Life Stud. 2015; 5(2): 33-53. doi: 10.19154/njwls.v5i2.4792.

44) Bull T. Work life and mental well-being: Single and coupled employed mothers in Southern Europe and Scandinavia. Glob Health Promot. 2009; 16(3): 6-16. doi: 10.1177/1757975909339764. PMID: 19773296.

45) De Moortel D, Vandenheede H, Vanroelen C. Contemporary employment arrangements and mental wellbeing in men and women across Europe: A cross-sectional study. Int J Equity Health. 2014; 13(1): 90. doi: 10.1186/s12939-014-0090-6. PMID: 25348746, PMCID: PMC4219120.

46) Rajgopal T. Mental well-being at the workplace. Indian J Occup Environ Med. 2010; 14(3): 63-5. doi: 10.4103/0019-5278.75691. PMID: 21461156, PMCID: PMC3062016.

47) Black SV, Cooper R, Martin KR, Brage S, Kuh D, Stafford M. Physical Activity and Mental Well-being in a Cohort Aged 60-64 Years. Am J Prev Med. 2015; 49(2): 172-80. doi: 10.1016/j.amepre.2015.03.009. PMID: 26070782, PMCID: PMC4518501. 